\title{
Rising to the Ebola challenge, again
}

\author{
The second outbreak of Ebola virus disease in recent months in the Democratic Republic of the Congo will test local \\ and international responses. Fortunately, the experience gained since the West African outbreak of 2013-2016 \\ means that we are better prepared to meet the challenge than ever before.
}

\begin{abstract}
$\mathrm{n}$ a cruel game of public health 'whacka-mole, just as the ninth outbreak of Ebola virus disease to hit the Democratic Republic of the Congo (DRC) was ending following a concerted response from local teams, international partners and donors, a new outbreak is beginning to unfold in the east of the country. A cluster of suspected cases of acute haemorrhagic fever in the North Kivu Province was reported to the Ministry of Health of the DRC on 28 July and four out of six samples tested positive for Ebola virus. Further investigation identified earlier sporadic deaths that were likely part of the same cluster and, at the time of writing, 30 Ebola virus disease cases have been confirmed, with more than 85 other probable or suspected cases. Genetic
\end{abstract} analyses have identified Zaire ebolavirus (EBOV) as the cause of the outbreak, as was the case with the earlier outbreak in the Équateur Province, although sequence data indicates that the two are not closely linked. This is the first time that EBOV has been found in this part of the DRC, and of particular concern is the confirmed case in the city of Beni, which has a population of around 350,000 and is well linked to other major population centres. Even more worrying is that this tenth outbreak is unfolding in a conflict zone with over one million displaced people and many active insurgent groups that could severely impede efforts to tackle the outbreak.

Ironically, the situation would be more bleak were it not for the recent outbreak in the west of the DRC, which has allowed the response to start on the front foot with experienced personnel and facilities already in place. For example, the Institut National de Recherche Biomédicale in Kinshasa, DRC, has established the local expertise necessary to carry out the initial analyses of patient samples, enabling swift identification of EBOV during the current and previous outbreak. Indeed, since the West African outbreak of 2013-2016, which caught national and international health bodies napping (S. Moon et al. Lancet 386, 2204-2221; 2015), there are clear signs that lessons have been learned, not just with improvements in local infrastructure but also in the speed with which an international response is mounted and in the new therapeutic tools being developed.

The Équateur Province outbreak, which officially ended on 24 July, just days before the first North Kivu cluster was reported, saw 54 confirmed cases and 33 fatalities low figures in comparison to the $\sim 29,000$ suspected cases and $\sim 11,000$ deaths during the West African outbreak a few years before. While there are many factors that affect the course and scale of a disease outbreak, the speed with which the WHO responded to the outbreak declaration on 8 May, releasing US $\$ 2$ million from its Contingency Fund for Emergencies within hours and deploying additional personnel to work alongside the health workers in the DRC, will have been a major factor in limiting the spread of infection. Also important was the deployment of the experimental rVSV-ZEBOV Ebola vaccine, which was used in the Équateur Province to vaccinate a ring of people around each infected individual and was seemingly effective in curtailing the outbreak. Over 3,000 doses of the vaccine remained available in the DRC as of the beginning of August (https://go.nature.com/2OWfZO9), and vaccination is underway in North Kivu, beginning with front-line health workers who had been in contact with confirmed EBOV infection cases. There have been doubts as to whether a ring vaccination strategy would be possible in North Kivu, given the impact of the security situation on the ability to trace individuals who have been in contact with those infected. The alternative, as noted by Peter Salama, head of the WHO Health Emergencies Programme, would be to target individuals for vaccination based on geography (that is, blanket vaccination of an entire village rather than just those villagers who have been in contact with an infected individual), which will increase demand on available rVSV-ZEBOV stocks (https:// go.nature.com $/ 2 \mathrm{vNjHky}$ ). Other challenges associated with outbreaks in areas affected by conflict will also have to be overcome, including maintaining medical support and surveillance in the face of a potentially diminished local health infrastructure, containing potential disease spread by displaced individuals, and guaranteeing safety for healthcare workers.

The North Kivu outbreak also provides an opportunity to test several additional experimental drugs and monoclonal antibodies to treat infected individuals. Although available during the previous outbreak, the speed with which the outbreak was brought under control outpaced the review process undertaken by the DRC's scientific and ethical committees, and the treatments were not, in the end, needed (https://go.nature.com/2ORaWyy). Ensuring that the review processes for the use of such experimental therapies on a compassionate basis is as rapid as possible will not only allow experimental therapies to be tested in outbreaks that are quickly brought under control, but could prove pivotal in saving lives in situations where vaccination approaches are impeded and case numbers are high.

Once the current outbreak is under control it will be important to assess and build on what has been learned, and to support local resources and technical expertise in public health laboratories and outbreak response teams. Maintaining vigilance and levels of preparedness between outbreaks is costly, but essential. As with fire-control measures, it is necessary to prepare defences against worst-case scenarios in the hope that they never have to be called on. While we encroach everfurther into the habitats of the reservoir hosts of putative zoonotic agents, failing to properly invest in prevention, early warning and rapid-response capabilities for emerging and re-emerging infectious diseases is a false economy that, in the long run, will have significantly greater economic and human costs. Similarly, ensuring that mechanisms are in place to fund research and development of therapeutics that, in an ideal future, will be left on the shelf is often a difficult proposition but is one that requires continued commitment.

Published online: 16 August 2018 https://doi.org/10.1038/s41564-018-0243-2 